\title{
Review of: "Genomic features underlie the co-option of SVA transposons as cis-regulatory elements in human pluripotent stem cells"
}

Ioannis Georgiou ${ }^{1}$

1 University of Ioannina

Potential competing interests: The author(s) declared that no potential competing interests exist.

Review: Genomic features underlie the co-option of SVA transposons as cisregulatory elements in human pluripotent stem cells

The manuscript by S.M. Barnada et. al. is a very interesting contemporary report combining advanced technology/molecular methods with bioinformatic tools, from the stage of the experimental design up to the stage of data analysis and presentation.

The research group chose to study the TE family of SVAs and elucidate their contribution in the regulation of nearby genes, either as vectors of regulatory elements, or as consensus sequences evolved to exploit regulatory factors for their activation.

Their work hypothesis is sound and clear, as the group is dedicated to the study of TEs and SVAs and attempts to answer fundamental biological questions related to their genomic functions. The molecular methods are also the adequate ones to experimentally approach the questions raised and have been applied in parallel with appropriate bioinformatic analysis as the results emerged and gradually answers were provided.

As the issue of the emergence and function of TEs originating from all classes and families is becoming hot, classic, and modern philosophy is put forward to extend the dimensions of the findings. Jacque Monod, the father of modern philosophical approach in biology introduced the materialistic idea of chance and necessity in evolution. Data are accumulating, and this study has conveyed even more data to the literature, sufficient to speculate whether SVA-Es and SVA-Fs that are shown to be preferentially associated with YY1/OCT4 consensuses and other sequences, have evolved in the human genome to coopt as cis regulatory elements.

A previous bioinformatic report (P. Ladias et al. 2017, J. Mol. Biol.) has shown that there is a preference for certain recombination related sequences with individual TE classes and families. Therefore, TEs as mentioned above, are either vectors of functional elements, by chance, or preexisting consensus sequences evolved overtime within SVAs to exploit regulatory factors for their activation.

In conclusion the elegant study by S.M. Barnada et. al. is a brilliant new addition to the study of TEs and 
SVAs, presenting more relevant answers to the scholars involved in the field, as well as is putting more research questions for future experiments. 